\title{
Treatment of a densely fused fissure during thoracoscopic right middle lobectomy ${ }^{*}$
}

\author{
Hiroyuki Koga $^{1 \#}$, Kenji Suzuki ${ }^{2}$, Tadaharu Okazaki ${ }^{1}$, Kinya Nishimura ${ }^{3}$, Geoffrey J. Lane ${ }^{1}$, \\ Atsuyuki Yamataka ${ }^{1}$ \\ ${ }^{1}$ Department of Pediatric General and Urogenital Surgery, Juntendo University School of Medicine, Tokyo, Japan \\ ${ }^{2}$ Department of Thoracic General Surgery, Juntendo University School of Medicine, Tokyo, Japan \\ ${ }^{3}$ Department of Anesthesiology, Juntendo University School of Medicine, Tokyo, Japan \\ Email: "
}

Received 4 January 2013; revised 6 February 2013; accepted 15 February 2013

Copyright ( 2013 Hiroyuki Koga et al. This is an open access article distributed under the Creative Commons Attribution License, which permits unrestricted use, distribution, and reproduction in any medium, provided the original work is properly cited.

\begin{abstract}
Aim: To report a case of fused fissure between the right upper and middle lobes that we treated using a surgical stapler rather than a Ligasure device for the benefit of creating a better division between the right upper and middle lobes and to effectively seal the lung parenchyma. Case: A 2-year-old girl with congenital cystic adenomatoid malformation of the right middle lobe (RML) was referred to our institution for further management after a series of infections. The vein of the RML, which drains into the superior pulmonary vein, was isolated and divided using endoclips. The bronchus was then exposed and divided using endo-clips. The arteries of the RML could be identified and ligated, allowing a line demarcating the major fissure to be identified and dissected. A stapler device was then used to seal the lung parenchyma and create a division between the right upper and middle lobes. She is currently well after follow-up of 16 months, with no episodes of respiratory distress or recurrence of symptoms. Conclusion: Our technique for dividing the pulmonary vein, then the bronchus, then the pulmonary artery and finally the fused fissure is safe and could be applied whenever fused fissures are encountered during thoracoscopic pulmonary lobectomy.
\end{abstract}

Keywords: Thoracoscopic Surgery; Pulmonary Lobectomy; Fused Fissure; Surgical Stapler

\footnotetext{
*This paper will be presented at the 20th annual congress of the International Pediatric Endosurgery Group, May 4-7, 2011 in Prague, Czech Republic.

"Corresponding author.
}

\section{INTRODUCTION}

With advancements in endoscopic technology and technique, thoracoscopic surgery is being used more commonly for the diagnosis and treatment of thoracic conditions in pediatrics. However, thoracoscopic pulmonary lobectomy can be difficult $[1,2]$. During major pulmonary resection using thoracoscopy, a Ligasure device (Valley lab, Boulder, CO) or bipolar sealing device is commonly used to seal the cut lung parenchyma and fissure surfaces [3]. However, in cases where a fissure is fused so strongly that it cannot even be identified, there is a risk for air leakage after major pulmonary resection. We report a case with a fused fissure between the right upper and middle lobes, where a surgical stapler was used instead of a Ligasure device since it was considered to be better for creating a division between the fused lobes and sealing the lung parenchyma.

\section{CASE REPORT}

A 2-year-old girl with congenital cystic adenomatoid malformation (CCAM) of the right middle lobe (RML) was referred to our institution for further management after a series of infections (Figure 1).

\subsection{Surgical Technique}

The patient was positioned in the lateral decubitus position under general anesthesia with single lung ventilation. The lateral decubitus position was used because it allows access to the hilum both anteriorly and posteriorly. For thoracoscopy, the surgeon and assistant stand in front of the patient and view a monitor behind the patient. A 5 $\mathrm{mm}$ port was placed in the fifth intercostal space in the anterior axillary line using Hasson's open technique to 


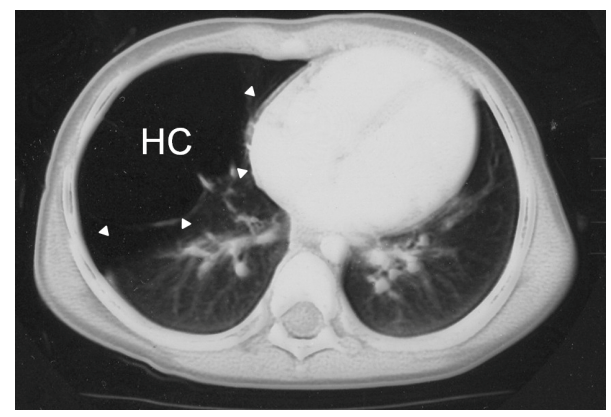

Figure 1. Computed tomography scan of the chest showing huge cystic lesion (HC: arrows) replacing the normal right middle lobe.

determine the position of the fissure and to evaluate the general condition of the lung parenchyma. Low flow $(0.5$ - $1.0 \mathrm{~L} / \mathrm{min}$ ) and low pressure $(4-6 \mathrm{mmHg}$ ) carbon dioxide was insufflated to collapse the lung. A $5 \mathrm{~mm}$ scope (less than 30 degrees) was used to determine the position of the major fissure and lung parenchyma and 4 other working ports were placed to allow optimal access. A Ligasure device was used to decompress multiple huge cysts, causing the RML to collapse completely. Upon close examination, the fissures of the right lung were found to be fused. In particular, the minor fissure was so densely fused it could not be distinguished at all. Firstly, the vein of the RML, which drains into the superior pulmonary vein, was isolated and divided using clips such as Endo-clips (Tyco Healthcare, CT, USA), or Hemolock-clips (Teleflex Medical, NC, USA). The bronchus was then exposed, encircled with a thick silk tie as a traction suture, and divided using Endo-clips by applying traction and counter traction on the silk tie. Finally, the arteries of the RML were identified and ligated using Endo-clips (Figure 2). By doing so, a line demarcating the major fissure could be identified and dissected using a stapling device, Echelon-flex (Ethicon Endo-surgery; Johnson \& Johnson, Cincinnati, OH, USA) used to seal the lung parenchyma and create a division between the right upper and middle lobes.

Currently after follow-up of 16 months, she is well, with no episodes of respiratory distress or recurrence of symptoms.

\section{DISCUSSION}

Rodgers first reported the use of thoracoscopy in the mid to late 1970s [4,5], and it has come to be used safely and efficiently for treating a number of pulmonary lesions [4] in the pediatric population. Now, thoracoscopic pulmonary lobectomy is performed in all age groups from neonates to adolescents, and is regarded as the treatment of choice at many institutions. However, treatment of major vessels and the bronchus has been challenging. Initially, a mini-thoracotomy was performed and a com-

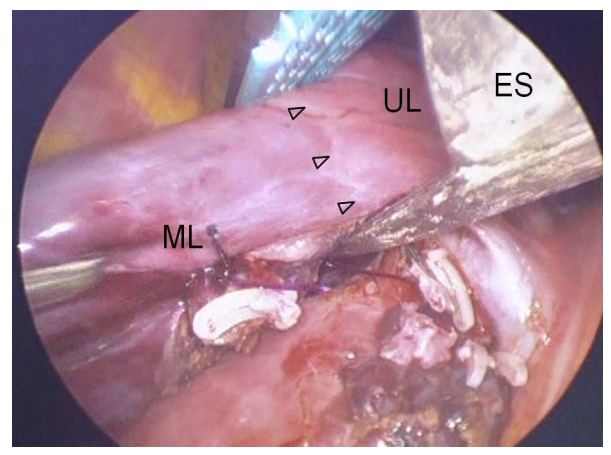

Figure 2. A line demarcating the major fissure (Arrows) could be identified with one continuous staple line, dissected and Endo stapler device (ES), used to seal the lung parenchyma and create a division between the right upper (UL) and middle lobes (ML).

bination of suture ligation and standard stapling was used. Over time, with technical improvement, such as development of the Ligasure device, pulmonary vessels could be treated completely using thoracoscopy, without thoracotomy.

To the best of our knowledge, there are no reports in the literature about the treatment of a densely fused fissure during thoracoscopic right middle lobectomy in a child. Rothenberg's report is the largest study dealing with minimally invasive lung resection, and he used a Ligasure device to compress, seal, and divide the lung where a fissure should have been. They reported that if the lung parenchyma was thick, it could even be done in layers, taking sequential bites of lung ${ }^{3}$. Incremental bites were made into the parenchyma at the suspected fissure site, then the Ligasure device was used to seal and divide the lobes. Such incremental bites were continued until the pulmonary vessels were exposed. However, he also described that there was often a slow, steady ooze from the fragile parenchyma even when a Ligasure device was used [6]. This conventional approach of exposing the pulmonary artery by dividing lung parenchyma overlying the artery within the fused fissure may have a risk for persistence air leak after pulmonary lobectomy, compared with our technique. In cases requiring pulmonary lobectomy with densely fused fissures, a Ligasure device may actually be unsuitable for dividing the fissure, although it is highly valued for other pulmonary surgery. Namely, the pulmonary vein and the bronchus of the RML are divided. This is followed by division of the RML artery, which lies just posterior to the divided bronchus. The fissure then is divided by an endo-stapler. This contributes to reduce the incidence of persistent postoperative air leak because there is no dissection of the fissure performed to expose the pulmonary artery. The incidence of persistence air leak after pulmonary lobectomy ranges from $8 \%$ to $26 \%$ [7-9]. Interestingly, 
the majority of patients with persistent air leak have persistent parenchymal alveolar air leak rather than a bronchopleural fistula due to bronchial stump dehiscence [10]. Dissection of a fused fissure for exposing the pulmonary artery during right upper pulmonary lobectomy has been suggested as contributing to the persistent postoperative air leak $[11,12]$. In fact, a fissure divided by stapling has less air leak, increased probability that the leak will resolve without an increase in morbidity and mortality when compared with more traditional techniques $[11,12]$.

To make any recommendation, we need to perform a significantly larger number of procedures. Also, our technique required far more staples than the Ligasure technique. As one staple cartridge costs approximately $\$ 250$ in Japan, the cost of a fused fissure could be up to $\$ 500$ higher. However, there was no incidence of postoperative air leakage. Indirectly, this will cut costs because hospitalization is shorter and fewer medications are required.

\section{CONCLUSION}

Our technique for dividing the pulmonary vein, the bronchus, and the pulmonary artery allows a fused fissure to be treated efficiently, enhancing the safety of thoracoscopic pulmonary lobectomy.

\section{ACKNOWLEDGEMENTS}

This study was supported in part by a Grant-in-Aide (S0991013) from the Ministry of Education, Culture, Sport, Science, and Technology, Japan (MEXT) through the Foundation for Strategic Research Projects in Private Universities.

\section{REFERENCES}

[1] Mack, M.J., Scruggs, G.R., Kelly, K.M., Shennib, H. and Landreneau, R.J. (1997) Video-assisted thoracic surgery: Has technology found its place? The Annals of Thoracic Surgery, 64, 211-215. doi:10.1016/S0003-4975(97)00247-6

[2] Yim, A.P., Landreneau, R.J., Izzat, M.B., Fung, A.L. and Wan, S. (1998) Is video-assisted thoracoscopic lobectomy a unified approach? The Annals of Thoracic Sur- gery, 66, 1155-1158. doi:10.1016/S0003-4975(98)00622-5

[3] Rothenberg, S.S. (2007) Thoracoscopic pulmonary surgery. Seminars in Pediatric Surgery, 16, 231-237. doi:10.1053/j.sempedsurg.2007.06.004

[4] Rothenberg, S.S. (2008) First decade's experience with thoracoscopic lobectomy in infants and children. Journal of Pediatric Surgery, 43, 40-44. doi:10.1016/j.jpedsurg.2007.09.015

[5] Rodgers, B.M., Moazam, F. and Talbert, J.L. (1979) Thoracoscopy in children. Annals of Surgery, 189, 176-180. doi:10.1097/00000658-197902000-00008

[6] Rothenberg, S.S., Kuenzler, K.A. and Middlesworth, W. (2009) Thoracoscopic lobectomy for severe bronchiectasis in children. Journal of Laparoendoscopic \& Advanced Surgical Techniques. Part A, 19, 555-557. doi:10.1089/lap.2009.0093

[7] Brunelli, A., Monteverde, M., Borri, A., Salati, M., Marasco, R.D. and Fianchini, A. (2004) Predictors of prolonged air leak after pulmonary lobectomy. The Annals of Thoracic Surgery, 77, 1205-1210. doi:10.1016/j.athoracsur.2003.10.082

[8] Abolhoda, A., Liu, D., Brooks, A. and Burt, M. (1998) Prolonged air leak following radical upper lobectomy: An analysis of incidence and possible risk factors. Chest, 113, 1507-1510. doi:10.1378/chest.113.6.1507

[9] Allen, M.S., Darling, G.E., Pechet, T.T., et al. (2006) Morbidity and mortality of major pulmonary resections in patients with early-stage lung cancer: initial results of the randomized, prospective ACOSOG Z0030 trial. The Annals of Thoracic Surgery, 81, 1013-1020. doi:10.1016/j.athoracsur.2005.06.066

[10] Rice, T.W., Okereke, I.C. and Blackstone, E.H. (2002) Persistent air-leak following pulmonary resection. Chest Surgery Clinics of North America, 12, 529-539. doi:10.1016/S1052-3359(02)00022-4

[11] Ng, T., Ryder, B.A., Machan, J.T. and Cioffi, W.G. (2010) Decreasing the incidence of prolonged air leak after right upper lobectomy with the anterior fissureless technique. The Journal of Thoracic and Cardiovascular Surgery, 139, 1007-1011. doi:10.1016/j.jtcvs.2009.07.023

[12] Nomori, H., Ohtsuka, T., Horio, H., Naruke, T. and Suemasu, K. (2003) Thoracoscopic lobectomy for lung cancer with a largely fused fissure. Chest, 123, 619-622. doi:10.1378/chest.123.2.619 